ORNL/TM-2007/157

\title{
Reactor Technology Options Study for Near-Term Deployment of GNEP Grid-Appropriate Reactors
}

\author{
D. T. Ingersoll \\ Nuclear Technology Programs Office \\ W. P. Poore, III \\ Nuclear Science and Technology Division
}

Date Published: September 26, 2007

Prepared by the OAK RIDGE NATIONAL LABORATORY

Oak Ridge, Tennessee 37831-6283 managed by UT-BATTELLE, LLC

for the

U.S. DEPARTMENT OF ENERGY under contract DE-AC05-00OR22725 



\section{CONTENTS}

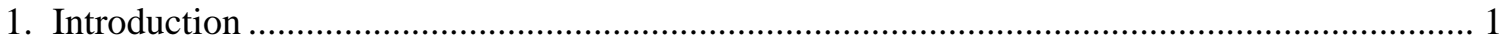

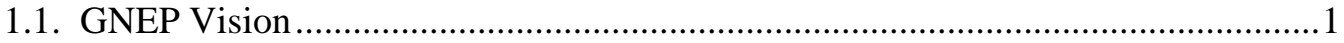

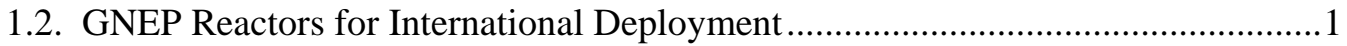

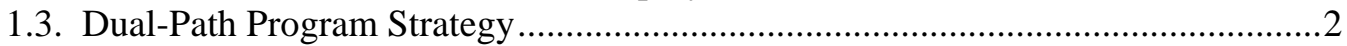

1.4. Near-Term Reactor Technology Options Study .....................................................

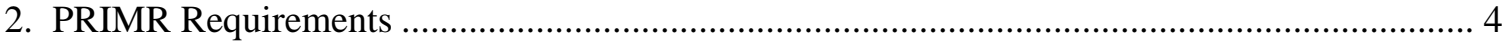

2.1. Plant Output Capacity ......................................................................................

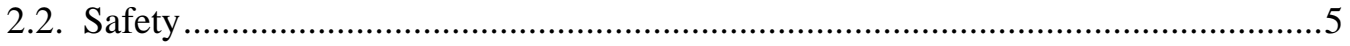

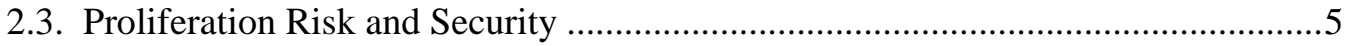

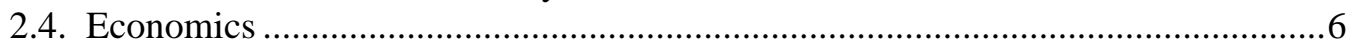

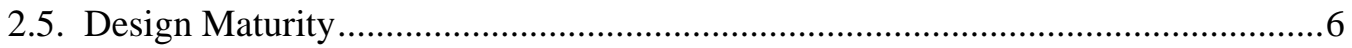

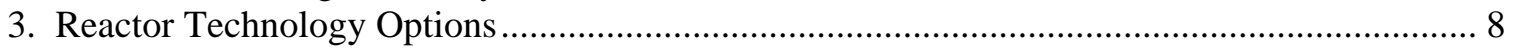

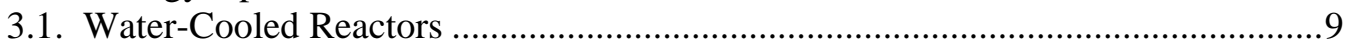

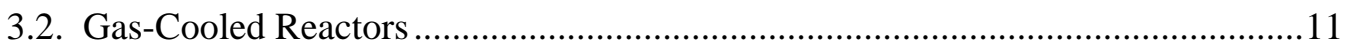

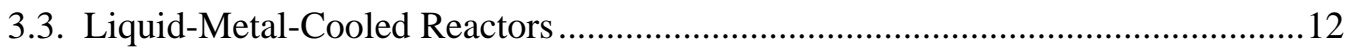



4. Evaluation of Technology Maturity for Near-Term Deployment .......................................... 17

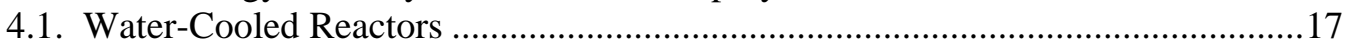



4.3. Liquid-Metal-Cooled Reactors ............................................................................ 18

4.4. Molten-Salt-Cooled Reactors ..........................................................................20

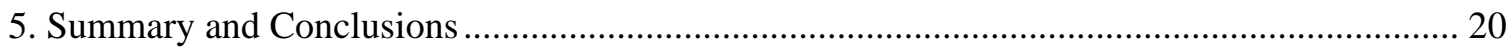

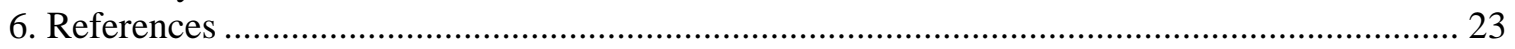





\section{LIST OF TABLES}

Table

1

2
Page

Summary of Technology Readiness Levels for measuring technical maturity ..............7

Maturity indicators for primary reactor types …..................................................21 



\section{LIST OF FIGURES}

Figure

1

2

3

4

5

6
Size distribution of electrical generating plants of all fuel types recorded worldwide.

Page

Diagram of integral primary system reactor design................................................10

Model of prismatic VHTR being developed for the Generation IV program...........11

Model of sodium-cooled ARR concept ...............................................................13

Model of SSTAR lead-cooled reactor concept ......................................................14

Diagram of molten-salt-cooled AHTR concept................................................16 



\section{Introduction}

\subsection{GNEP Vision}

World energy demand is projected to significantly increase over the coming decades. The International Energy Agency projects that electricity demand will increase 50\% by 2015 and double by 2030, with most of the increase coming in developing countries as they experience double-digit rates of economic growth and seek to improve their standards of living. Energy is the necessary driver for human development, and the demand for energy in these countries will be met using whatever production technologies are available. Recognizing this inevitable energy demand and its implications for the United States, the U.S. National Security Strategy has proposed the Global Nuclear Energy Partnership (GNEP) to: “...work with other nations to develop and deploy advanced nuclear recycling and reactor technologies. This initiative will help provide reliable, emission-free energy with less of the waste burden of older technologies and without making available separated plutonium that could be used by rogue states or terrorists for nuclear weapons. These new technologies will make possible a dramatic expansion of safe, clean nuclear energy to help meet the growing global energy demand."1

In other words, GNEP seeks to create an international regime to support large-scale growth in the worldwide use of nuclear energy without increasing the risk of nuclear weapon proliferation. This global expansion of nuclear power is strategically important to the United States for several reasons, including the following:

- National security, by reducing the competition and potential for conflict over increasingly scarce fossil energy resources

- Economic security, by helping maintain stable prices for nonrenewable resources such as oil, gas, and coal

- Environmental security, by replacing or off-setting large-scale burning of greenhouse gas-emitting fuels for electricity production

- Regaining technical leadership, through deployment of innovative U.S. technology-based reactors

Fully meeting the GNEP vision may require the deployment of thousands of reactors during the next century in dozens of countries, many of which are in the developing world where nuclear energy is not used currently. Such a large-scale deployment will have significant implications related to both fuel supply and spent fuel/waste management, both domestically and worldwide. Consequently, GNEP must address the development and demonstration of proliferation-resistant technologies to ensure both a safe and sustainable nuclear fuel cycle, and reactor designs that are appropriate for the range of needs across the global community. The focus of this report is the latter need, that is, the development and demonstration of proliferation-resistant reactors that are well matched to the needs and capabilities of developing countries.

\subsection{GNEP Reactors for International Deployment}

To date, the world's major reactor vendors have targeted markets in developed countries and currently offer designs that have large power outputs (1000-1700 MWe). However, these large reactors are unsuitable for many developing countries for several reasons, including the following.

- Many developing countries have limited electric grid capacity that cannot accommodate a single power plant with output approaching or exceeding 1000 MWe. Also, the grid in 
some countries is localized in a few isolated population centers with minimal interconnections. This situation favors the use of smaller power plants sited at geographically separated locations.

- Nuclear power plants traditionally have a large capital cost relative to fossil power plants, which creates an additional barrier to choosing nuclear power. By virtue of their reduced size and complexity, smaller-sized nuclear plants will have a lower capital cost per plant and shorter construction time. Thus the initial power unit can be generating revenue before the second and third units are constructed, thus reducing the maximum capital outlay for the combined generating capacity. This is especially important for developing economies, which typically have limited availability of capital funds.

- Because of the lower power levels of small or medium-sized nuclear plants, countries have more flexibility to install generating capacity in smaller increments that better match their rate of power demand and economic growth.

- The reduced power levels allow greater use of passive safety systems and plant simplifications, such as natural circulation of the primary coolant. These features enhance the safety and reliability of the nuclear power facility, which is especially advantageous in countries that have limited nuclear experience and trained workforces. The enhanced safety also allows the plants to be sited closer to population centers, thus reducing the cost of transmission lines or heat transport lines.

Several countries, including France, Russia, Japan, Korea, South Africa, India, and Argentina, have already recognized the global market need for smaller-sized nuclear power plants and are moving forward aggressively with the development of small and medium-sized reactors (SMR). For example, Russia is actively pursuing deployment of its KLT-40S floating reactor in Indonesia and is pursuing nuclear cooperation with numerous developing countries such as Egypt, Chile, and Namibia. ${ }^{2}$ Also, India has announced its intent to market its 220-MWe Pressurized Heavy Water Reactor (PHWR) in Southeast Asia, ${ }^{3}$ and Argentina and the Republic of Korea are actively developing small-reactor concepts specifically targeted for export to developing countries. Japan has been pursuing the export of small, simplified reactors to remote locations such as Galena, Alaska.

In the United States, several design studies for smaller-sized nuclear power plants are being pursued at various levels of support. It is vital that the United States accelerate the development of suitable reactor systems in order to enable the U.S. nuclear industry to compete effectively in this growing international market and ensure the safety, security, and proliferation-resistant characteristics of the reactors that will be deployed internationally. The successful deployment of these reactors, which we designate as "proliferation-resistant international modular reactors" (PRIMR), together with the GNEP policy of reliable fuel services, will provide an attractive energy solution to many countries and will serve to eliminate the need for them to develop the more proliferation-vulnerable parts of the nuclear fuel cycle, thereby achieving key GNEP strategic objectives.

\subsection{Dual-Path Program Strategy}

Because it is ultimately the responsibility of private industry to develop and market commercial nuclear power plants, the role of the U.S. Department of Energy (DOE) program will be to pave the way for U.S. industry to effectively compete in the international market by helping to remove various barriers for deployment and to accelerate development and demonstration of new technologies or innovative designs. To accomplish this, a dual-path approach has been formulated for PRIMR development and demonstration. The first path provides a fast-track implementation that strives to have a near-term plant design ready for deployment by 2015 . The 
date of 2015 was chosen to be responsive to the existing international demand for increased power. Additionally, this fast-track deployment strategy will better allow the United States to

- influence other supplier countries working to deploy similar reactors to meet GNEP strategic objectives,

- facilitate U.S. industry participation and competitiveness in the rapidly emerging nuclear market, and

- provide near-term credibility in meeting key GNEP objectives.

Pursuing this fast track will serve to identify and help resolve related infrastructure and regulatory needs for deployment of PRIMR systems in developing countries, such as plant licensing, workforce education and training, international agreements, international safeguards, and physical protection.

The urgency of initiating the development and demonstration of near-term PRIMR systems was identified in recent studies funded by DOE, ${ }^{4,5}$ which highlighted the realities of global-energydemand growth and the accelerating level of activities by several other reactor-supplier countries. The most recent study concluded that "if GNEP is to succeed in its global non-proliferation goals, it must 1) take SMRs seriously into account, and 2) recognize that significant technology and commercial developments are already underway."

The second path in the dual-path strategy focuses on accelerating reactor technology developments that are needed to deploy next-generation PRIMR designs suitable for a broader global market. These designs will offer further enhancements in plant safety, proliferation resistance, and security. They also will provide enhancements in features and capabilities such as improved economics, operational simplifications, extended core life, and factory fabricability. The next-generation PRIMR designs will build on the successful resolution of critical infrastructure issues for the near-term PRIMR system and will involve the development of more robust reactor technologies. Because of the $R \& D$ needed to achieve these performance objectives, the next-generation PRIMR is targeted for a deployment date of 2030.

\subsection{Near-Term Reactor Technology Options Study}

The dual-path strategy for PRIMR development allows the program to move forward quickly but requires a reactor technology that is sufficiently mature to allow for the deployment of a new design by roughly 2015. The selected system design must also satisfy several constraints and requirements imposed by top-level GNEP goals, that is, safety, proliferation resistance, and safeguards and security. These requirements are being developed cooperatively with international partners and will include the development of specific criteria and an evaluation process so that specific PRIMR designs can be evaluated for eventual deployment.

The first step in the process of selecting a reactor design for near-term deployment is the evaluation of basic reactor technologies with respect to their readiness or maturity. This evaluation has been performed and is presented in the following sections. A brief description of top-level constraints and requirements with an emphasis on technology maturity is given in Section 2. Section 3 provides a brief description of the candidate reactor technologies, which are categorized by coolant type. An assessment of the maturity of these technologies is given in Section 4, which provides the basis for selection of a near-term PRIMR technology. 


\section{PRIMR Requirements}

For successful deployment within the GNEP context, a PRIMR system must satisfy several constraints and requirements. As examples, the reactor should be

- $\quad$ sized for market conditions in developing countries where the electrical grid and industrial infrastructure are at varying stages of maturity;

- sufficiently safe, proliferation resistant, and secure to meet the primary goals of GNEP; and

- economically competitive with alternative sources of energy in the country of deployment.

Also, reactor designs must include considerable U.S. industry participation if significant investments are to be made by the U.S. Government. Additionally, the near-term PRIMR must be sufficiently mature so as to be ready for deployment by 2015. This imposes a number of constraints on the selection of reactor technology and design in terms of demonstrated performance, licensing timelines, industry readiness, etc. Brief descriptions of these top-level requirements are presented below. For the fast-track phase of the dual-path strategy, emphasis is given to technical maturity because this characteristic will be a key discriminator in selecting a near-term PRIMR design. For the longer-term track, more aggressive performance targets will be developed and used to evaluate candidate designs.

\subsection{Plant Output Capacity}

As an accepted rule of thumb, individual plant size should be no more than $10-15 \%$ of the total electricity transmission grid capacity in order to ensure grid stability. Many developing countries currently have total grid capacities of less than $5 \mathrm{GWe}$; hence, plants with power ratings below 500 MWe are most suitable. Figure 1 shows the size distribution for all types of electrical generation plants surveyed worldwide. ${ }^{6}$ The data show that over $85 \%$ of the plants have outputs below $500 \mathrm{MWe}$, which provides further indication that smaller-sized nuclear plants may be better accommodated on existing grids. Even in developed countries that have a very large total grid capacity, the grids in some regional locations are not able to support new electrical capacity in large increments because they are near saturation or have limited interconnections to the main grid.

Some niche markets may require more or less power per plant.

For example, the Alaskan city of Galena and the island of Tinian are both considering nuclear plants in the range of 10-20 MWe. Other factors that impact the preferred size of a

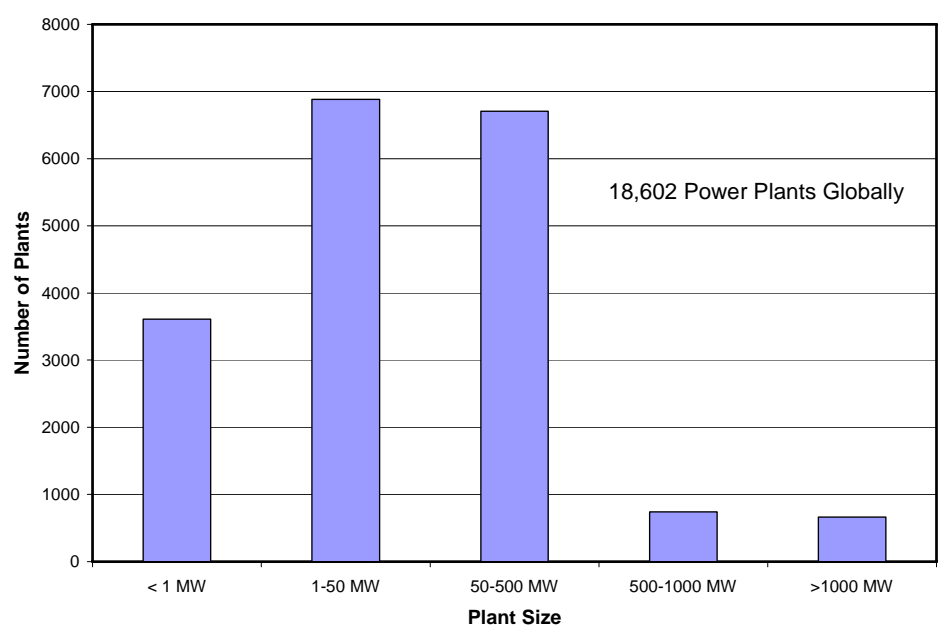

Fig. 1. Size distribution of electrical generating plants of all fuel types recorded worldwide. 
new plant include projected demand growth rate, construction cost, and cogeneration needs such as water desalination and district heating.

\subsection{Safety}

The safety of a nuclear power plant is a critical requirement but a complex characteristic to quantify. As was demonstrated with the Chernobyl accident, a severe nuclear accident anywhere in the world has substantial impact on the nuclear power industry everywhere. Therefore, a GNEP PRIMR system must meet internationally accepted safety standards regardless of which country supplies the plant. As the number of plants increases worldwide, it may be that even more stringent safety standards will be needed in order to ensure that the cumulative risk remains level.

All developed nuclear states have established regulatory organizations to ensure acceptable protection of the general public, and the authority of these organizations must be accounted for in the GNEP framework. At a minimum, it is expected that the design of a candidate PRIMR system must be approved by a qualified nuclear regulatory authority in the country of origin. For a U.S.-based PRIMR design, this requirement would be met by securing a Final Design Approval from the U.S. Nuclear Regulatory Commission (NRC). Additionally, it is assumed that the country where the reactor is deployed will be responsible for regulating construction and operation of the plant, so adequate education and training of regulatory staff in those countries will also be required.

\subsection{Proliferation Risk and Security}

A cornerstone of the GNEP vision is the expansion of nuclear energy without an increase in the risk of nuclear proliferation associated with civil nuclear activities. Like safety, proliferation resistance is a complex characteristic that is not easily quantified but requires a careful and comprehensive assessment of threats and multiple factors that determine the relative proliferation risk of a particular nuclear technology or fuel cycle architecture. Certain attributes of both the specific reactor facility and the broader fuel cycle architecture that supports it (e.g., fuel type, fuel transportation requirements, fuel processing technology) impact the overall proliferation risk. Proliferation risk can be assessed in terms of how the intrinsic and extrinsic attributes of a nuclear facility or fuel cycle architecture impede proliferation by a national actor (e.g., a non-nuclear weapon state that hosts the power plant). Physical protection risk associated with acquisition of nuclear materials or radiological sabotage by a subnational actor establishes a second and separate category of considerations for evaluating a proposed reactor design or fuel cycle system. Physical protection risk spotlights both national safeguards and security practices and facility design features that are intended to prevent successful terrorist attack or mitigate its consequences. It is essential to keep national proliferation and subnational physical protection risks separate and to consider the full national and international contexts within which a particular nuclear system is deployed.

With respect to commercial nuclear power, reactor operations are generally accepted to be less proliferation prone than uranium enrichment and spent fuel reprocessing operations. A key aspect of GNEP is to limit the further spread of enrichment and reprocessing activities through a new international fuel-cycle architecture that incorporates a "reliable fuel services" framework. This reliable fuel services framework will allow a country to choose the nuclear option to meet their electricity demand without the need to establish enrichment and reprocessing capabilities. For this to work effectively, PRIMR systems should use fuel forms where the fresh fuel supply and spent fuel disposition can be implemented within a reliable fuel services framework. This implies that the reactor must use fuel that is compatible with the fuel fabrication, fuel processing 
and disposition, and nuclear materials management technologies provided by the fuel supplier countries.

Taking a comprehensive approach to the evaluation proliferation resistance and physical protection risk helps assess the impact of specific reactor design features on overall proliferation and physical protection risk, and to take such risks into account when designing a facility. For example, heavy-water-moderated reactors have excellent "neutron economy" owing to the lowneutron-absorption characteristics of the moderator. These types of reactors allow the use of fuel with lower fissile enrichment, which reduces proliferation risk; however, the same feature of good neutron economy provides the reactor operator with the potential to produce weaponsusable material within in-core targets during normal power operation, thus increasing the plant's proliferation risk. The frequency of fresh fuel delivery and spent fuel discharge, as well as the proliferation and physical protection risk attributes of those nuclear materials, are among other factors that affect the total proliferation and physical protection risk associated with such reactors.

Of particular importance to proliferation risk is how effectively and efficiently a given reactor technology and its fuel can be safeguarded by the International Atomic Energy Agency (IAEA). Likewise, the extent to which the reactor design facilitates physical protection against theft or sabotage and/or mitigates the consequences of sabotage is of direct relevance to the security risks posed by the design with respect to subnational threats. Effective use of advanced international safeguards and domestic physical protection measures will be essential for PRIMR systems and will need to be incorporated early into the reactor design. At a minimum, international "safeguardability" and physical protection must meet current IAEA guidelines in INFCIRC/ $225^{8}$ and domestic physical protection requirements. How well a particular reactor design or concept facilitates international safeguards and effective physical protection is an important evaluation discriminator.

\subsection{Economics}

Competitive economics for electric power generation is important but may not be the overriding consideration when accounting for the customers' needs. These needs comprise more than cost alone and include reliability, convenience, security of future fuel supply, generation of local jobs, environmental compatibility, opportunities for affiliated local business, etc. Even so, capital and operating costs of the SMR should be kept as low as practical and comparable to alternative sources of power available in the country or region of use, which can vary by more than a factor of 10 for some parts of the world. Mass production, the application of advanced manufacturing technologies, and factory manufacture of standardized plants that are modularized for delivery and rapid site assembly are approaches that can offset the cost disadvantages normally associated with smaller plant sizes.

\subsection{Design Maturity}

Forty-two countries attended a workshop on the "Issues for the Introduction of Nuclear Power," which was sponsored by several countries that have mature nuclear power programs and hosted by the IAEA in December 2006. Over 70\% of the countries who participated in the workshop do not currently have nuclear power but are interested in pursuing the option. Subsequent to the workshop, several countries (Armenia, Jordan, Ghana, Namibia, Chile, Bahrain, Algeria, Egypt, Yemen, Malaysia, and Vietnam) have officially announced their intent to initiate or expand nuclear power in their country. Several of these countries cite the need to pursue the option aggressively in order to avoid serious power shortfalls by the middle of the next decade. 
The fast-track path of the planned dual-path program plan is intended to help meet this projected demand for new power generation worldwide, while also positioning the United States to better influence and benefit from the anticipated global growth of nuclear power. To have appropriate reactor plant designs ready for deployment by 2015, that is, ready to start construction, imposes a significant requirement regarding maturity of the design, including its fundamental technology, its ease of licensing, the extent of industry readiness, and the potential need for a pre-commercial demonstration unit. These criteria are described in more detail below.

2.5.1. Technology Maturity: In order to be ready for deployment by 2015, a near-term PRIMR system must be based substantially on proven reactor technology. For example, reactor designs that require new materials or fuel development are precluded for consideration as a nearterm PRIMR because the development and qualification cycle alone can take more than a decade. The National Aeronautics and Space Administration and the Department of Defense frequently use a nine-level Technology Readiness Level (TRL) scale to measure the maturity of technologies and systems for in-field use. The TRL scale adopted for use in GNEP is summarized in Table 1. It is clear that a near-term PRIMR system must fit substantially within the "proof-of-performance" category for the entire plant, including the reactor, power conversion system, and balance of plant systems. A limited number of individual components with lower TRLs may be included if the time to achieve proof-of-performance levels is acceptably short.

Table 1. Summary of Technology Readiness Levels for measuring technical maturity

\begin{tabular}{|c|c|c|}
\hline TRL & Function & Definition \\
\hline 1 & \multirow{3}{*}{ Concept Development } & Basic principles observed and reported \\
\hline 2 & & Technology concepts and/or applications formulated \\
\hline 3 & & $\begin{array}{l}\text { Analytical and experimental demonstration of critical function } \\
\text { and/or proof of concept }\end{array}$ \\
\hline 4 & \multirow{3}{*}{ Proof of Principle } & $\begin{array}{l}\text { Component and/or bench-scale validation in a laboratory } \\
\text { environment }\end{array}$ \\
\hline 5 & & $\begin{array}{l}\text { Component and/or breadboard validation in a relevant } \\
\text { environment }\end{array}$ \\
\hline 6 & & $\begin{array}{l}\text { System/subsystem model or prototype demonstration in } \\
\text { relevant environment }\end{array}$ \\
\hline 7 & \multirow{3}{*}{ Proof of Performance } & System prototype demonstration in prototypic environment \\
\hline 8 & & $\begin{array}{l}\text { Actual system completed and qualified through test and } \\
\text { demonstration }\end{array}$ \\
\hline 9 & & Actual system proven through successful operations \\
\hline
\end{tabular}

2.5.2. Licensing Readiness: For traditional or evolutionary reactor designs, Final Design Approval (FDA) by the NRC is expected to take up to 3 years and Design Certification (DC) may require and additional 1-2 years. Detailed design engineering will require 3-4 years to complete, and construction will require at least 3 years. Although some of these activities can overlap, the implication is that a new design must be ready to proceed to formal licensing no later than 2010 to be considered for near-term deployment. This allows only 3 years (2007-2009) for completion of the required preliminary design and safety analysis work, including supporting validation tests. 
Historically the NRC has licensed new reactor designs using 10 CFR Part 50 but now plans on using the 10 CFR Part 52 licensing framework. The significant benefit of Part 52 is that the previously separate construction permit and operating license are joined into a single combined operating license (COL). If the first near-term PRIMR unit is to be built in the United States and be ready for construction by 2015, it will need to be capable of being licensed using the current Part 50 process or the newer Part 52 process. If the first unit is constructed elsewhere, then it must be capable of timely licensing and approval by the regulatory authority in the country of deployment.

2.5.3. Industry Support: Another consideration for selecting a PRIMR technology for development and deployment is the ability of the nuclear industry to support the fabrication and operation of the reactor and balance of plant. In order to ensure timely and reliable availability of components and expertise, the chosen reactor technology must be able to draw from the existing industrial base but need not be restricted to U.S. industry. In fact, it is widely recognized that the construction of any new power plant, whether it be a GNEP PRIMR or a large Generation III plant, will require many of the components to be provided by foreign suppliers. Still, deployment of a PRIMR system would be a significant driver in reinvigorating the U.S. nuclear industry.

2.5.4. Prototype/Demonstration Unit: Because the near-term PRIMR will need to be based on proven reactor technology, a technology that requires the construction of a test unit prior to commercial deployment is precluded from consideration. The construction of a prototype or demonstration facility should also not be required; however, it may be desirable for nontechnology reasons to build a demonstration-type unit to facilitate other aspects of PRIMR deployment, such as licensing and workforce training. The preference is for the first PRIMR design to be capable of proceeding directly to construction of a first-of-a-kind (FOAK) commercial plant.

\section{Reactor Technology Options}

According to two recent IAEA reports, ${ }^{9,10}$ approximately 60 SMRs are being studied by various countries. These reactor designs have a diverse set of features and capabilities. Some are based on technologies and fuel cycles proven in commercial reactors used worldwide and are available for near-term consideration. For the longer term, design innovations using different fuel types, coolants, high-temperature materials, and fuel cycle strategies offer further benefits of enhanced economics, safety, safeguards, and proliferation resistance.

Reactor systems are typically categorized by their type of primary coolant system, including

- water-light and heavy,

- gas-carbon dioxide or helium,

- liquid metal—sodium, lead, or lead-bismuth, and

- molten salt — with or without dissolved fuel.

This chapter provides a brief summary of currently operating and advanced (evolutionary and revolutionary) reactor types based on these coolant technologies. Examples are given of new designs and concepts of special interest to GNEP. Specific aspects of the reactor types related to their suitability and readiness for near-term deployment are provided in Section 4. 


\subsection{Water-Cooled Reactors}

Water-cooled reactors make up about $95 \%$ of the commercial power reactors in operation worldwide. They come in a variety of design types, including pressurized water reactors (PWR), boiling water reactors (BWR), pressurized heavy-water reactors (PHWR), and water-cooled, graphite-moderated reactors (LWGR). Power demands, national economic growth, and economies of scale resulted in rapid growth in plant size following the introduction of smaller demonstration units; hence, most water-cooled reactors offered today exceed 1000-MWe capacity and some exceed 1500 MWe per unit. Hence, these reactors are used mostly in developed countries with large electric power grids, and these countries will likely continue to build large reactor units. Another design type, the integral primary system reactor (IPSR), is being developed by several countries (Argentina, France, Korea, and the United States) for small and medium-sized applications.

The currently operating designs rely on diversity and defense in depth to ensure safety under transient or accident conditions. There are multiple trains of multiple safety and control systems powered by safety-grade AC and DC power systems to shut down and cool the reactor for expected initiators, such accidents as loss of load, loss of offsite power, or loss of coolant. The newer designs, designated Generation III+, are generally less complex and make increased use of passive safety features in order to reduce construction and operating expenses.

Brief descriptions of major water-cooled reactor design types, and variations within each type, are provided below, followed by evolutionary water-cooled reactor designs.

3.1.1 Pressurized Water Reactors: Loop-type PWRs have been used for commercial power generation for over 40 years worldwide. About $70 \%$ of the world's operating power reactors are PWRs (67\% in the United States). Most commercial PWRs use low enriched uranium (LEU) fuel with less than $5 \mathrm{wt} \%{ }^{235} \mathrm{U}$. Some PWRs use mixed-oxide (MOX) fuel composed of uranium mixed with plutonium separated from used reactor fuel. Water coolant at high pressure, typically $15 \mathrm{MPa}$ (2200 psi), removes heat from the core with an exit temperature near $350^{\circ} \mathrm{C}$ and transfers it to a heat exchanger to boil water and make steam in a lower-pressure secondary system. The primary system water also serves as a neutron moderator to create a thermal neutron energy spectrum. The steam produced in the steam generator drives a turbine to generate electricity.

The CANDU reactor is a different type of PWR that uses heavy water instead of light water as the primary coolant and as the neutron moderator. Because heavy water does not absorb as many neutrons as light water, natural uranium may be used as a fuel, consequently avoiding a uranium enrichment infrastructure.

Currently operating PWRs use redundant and backup systems to cool the core in the case of plant operational transients, accidents, or emergencies. Advanced PWR designs, such as the Westinghouse AP1000, use passive safety systems instead of electric- or steam-powered safety systems to improve plant safety, reliability, and economics. The Russian VBER-300 is an example of a loop-type PWR that is being developed for the SMR market, as well as the 220-MWe Indian PHWR.

3.1.2. Boiling Water Reactors: Like PWRs, BWRs have been used for commercial nuclear power production for over 40 years. About $25 \%$ of the world's operating power reactors are BWRs (33\% in the United States). Commercial BWRs produce steam at saturated conditions in the reactor core. Moisture is separated from the steam before it leaves the reactor vessel on its way to the turbine, thus avoiding the large steam generator and pressurizer components required in PWRs.

As a variant of the BWR, there are 16 LWGR plants operating in Russia or Lithuania. These reactors permit water to boil in the core inside pressurized coolant tubes or channels. The 16 
LWGR plants have some design benefits, such as a low power density and on-line refueling; however, they were not designed to Western safety standards.

Currently operating BWRs use redundant and backup systems to cool the core in the case of plant operational transients, accidents, or emergencies. Advanced BWR designs, such as the General Electric ESBWR, use passive safety systems instead of electric- or steam-powered safety systems to improve plant safety, reliability, and economics. Examples of BWR designs that might be suitable for PRIMR application are the CM-BWR being developed by General Electric, Toshiba's CCR design, and Hitachi's DMS design.

3.1.3 Integral Primary System Reactors: A number of IPSR designs are being developed worldwide. They are characterized by integrating primary system components such as steam generators and the pressurizer into a single pressure vessel. ${ }^{11}$ Figure 2 gives a schematic of an IPSR vessel internals. ${ }^{12}$ Designs of this type are claimed to reduce or eliminate some accident sequence initiators so that the consequences of these accidents are precluded, consequently, improving safety. For example, since vessel failures are considered to be extremely improbable events, and the primary coolant system is wholly contained in the IPSR vessel, the potential for a large-break loss of primary coolant (LOCA) event is eliminated. These designs also benefit from improved natural circulation cooling of the core in the event of loss-of-flow accidents. Some designs of this type also incorporate high-pressure containments and have significant water inventories to mitigate the consequences of small- and medium-break LOCAs.

Some IPSR designs are based on conventional PWR conditions, that is, temperature, pressure, coolant volume management, coolant pumps, steam generators, etc. Some smaller designs operate at reduced temperature and pressure to improve safety margins, gain longer fuel cycles, eliminate active safety systems, and operate safely at full power under natural circulation. Several boiling water designs offering fullpower natural circulation capability and passive emergency systems have also been designed. Most designs use conventional uranium oxide fuel or are MOX capable. Materials requirements are also similar to conventional LWR technologies.

Because of its promise of increased safety, the IPSR design lends itself well to diverse applications, including deployment in developing countries. The first operating IPSR was the German nuclear research ship Otto Hahn, which used an IPSR for ship propulsion and electric power generation during its 10-year operational demonstration in the late 1960s and 1970s. The U.S.-based IRIS design (shown in Fig. 2), the Korean SMART design, and the Argentinean CAREM design are examples of IPSRs. These reactor designs, as well as other similar designs such as the Japanese IMR, are



Fig. 2. Diagram of integral primary system reactor design. 
described in a number of well-referenced compilations ${ }^{1,6,13,14}$ of reactor designs provided by the Generation IV International Forum and the IAEA. These compilations offer summaries of features, attributes, economics, etc., of many small and medium-sized LWRs.

\subsection{Gas-Cooled Reactors}

Several gas-cooled reactors (GCR) have been built and operated using either $\mathrm{CO}_{2}$ or helium gas to cool the reactor core. Commercial GCRs use a graphite neutron moderator, which absorbs fewer neutrons than water-moderated reactors. The reactor designs offer some safety and operational benefits over water-cooled reactors, such as a lower core power density and a high heat capacity core, which helps limit fuel temperatures following a loss-of-coolant accident. They also avoid steam pressurization events present with high-pressure water coolant and also steam/zirconium cladding chemical reactions that can release hydrogen gas under accident conditions. The gas coolant also enables the plant to operate at higher temperatures $\left(>600^{\circ} \mathrm{C}\right)$ and, therefore, yield higher thermal efficiency than water-cooled reactor plants. Unlike traditional PWRs and BWRs, many GCR designs have the capability of being refueled during full-power operation. While this provides some operational convenience and higher plant availability, the continuous fuel access and handling represents an increased proliferation risk.

The first-generation GCRs operated in the U.K. and France and used natural uranium metal fuel and magnesium or magnesium alloys for the cladding. Subsequent plants used low-enricheduranium (LEU) oxide fuel clad with stainless steel. Beginning in Germany in the late 1950s, a new generation of helium-cooled GCRs was developed that used graphite-coated particle fuel. A fuel particle consists of a uranium kernel surrounded by a porous pyrolytic carbon buffer layer, an isotropic pyrolytic carbon layer, a silicon carbide barrier layer, and another isotropic pyrolytic carbon layer. The uranium in the fuel kernel is typically enriched above the 5\% level of LWR fuels but below the $20 \%$ enrichment ceiling for LEU fuel. Sometimes referred to as "TRISO" fuel, the coated fuel particles provide a highly corrosion-resistant and almost impermeable fission product containment boundary under normal and accident conditions. They maintain their integrity to beyond $1700^{\circ} \mathrm{C}$, which is a higher temperature than anticipated during credible accidents scenarios. The coated fuel particles are then formed into fuel compacts that are surrounded by a graphite moderator, which typically take the form of hexagonal blocks (prismatic variant) or spherical balls (pebble-bed variant). These variants are discussed in more detail below.

3.2.1 Prismatic Block Reactors: The most recent GCR constructed in the United States was the Fort St. Vrain reactor. It used prismatic block, graphite fuel elements, a ${ }^{235}$ U-thorium fuel cycle, and helium coolant. Uranium and thorium carbide fuel particles were coated in layers of pyrolytic carbon and silicon carbide. Steam turbine-driven helium circulators with waterlubricated bearings were used to force helium coolant through the core with an outlet temperature of about $775^{\circ} \mathrm{C}$. The outlet helium was circulated through steam generators to produce steam to feed the turbine generator.

Currently the Japanese are operating a 30-MWt prismatic helium-cooled high-temperature test reactor (HTTR). The United States is working to develop designs for two different heliumcooled GCRs: one in cooperation with Russia for the consumption of excess weapons plutonium (GT-MHR) and one for the high-temperature production of hydrogen (VHTR). The latter design is being developed for the Next Generation Nuclear Plant (NGNP) project as part of the Generation IV program, and it is still undecided whether that reactor will use a prismatic moderator form or a pebble bed form. A model of the prismatic VHTR is shown in Fig. 3.

3.2.2 Pebble Bed Reactors: Three pebble bed reactors have been constructed and operated: the German AVR and THTR test reactors and the currently operating Chinese HTR-10. The first 
commercial pebble bed reactor is expected to be the 165-MWe Pebble Bed Modular Reactor (PBMR) that is being constructed in South Africa by Eskom. ${ }^{15}$ The PBMR uses fuel spheres (pebbles), each about $6 \mathrm{~cm}$ in diameter and consisting of an outer shell of graphite and an inner fuel zone of fuel particles - about 15,000 per pebble. Approximately 440,000 pebbles make up the reactor core; about $75 \%$ of the spheres contain fuel and $25 \%$ contain only graphite to provide additional neutron moderation.

One operational advantage of the pebble bed reactor is its ability to be refueled on-line, that is, during full-power operation. Individual pebbles are removed from the bottom of the reactor and checked for accumulated burnup of the fuel within the pebble. If it has not yet achieved the target burnup, it is recycled into the top of the pebble bed for another pass through the core region. This process allows each pebble to achieve maximum burnup and hence higher uranium utilization compared to reactors with batch refueling. As discussed previously, however, the on-line refueling increases access opportunities for fresh and spent fuel, which is a proliferation concern.

Eskom expects to sell a large number of PBMRs to the African and international markets. The Chinese, based on their experience with HTR-10, are actively developing a 200-MWe commercial-sized plant designated

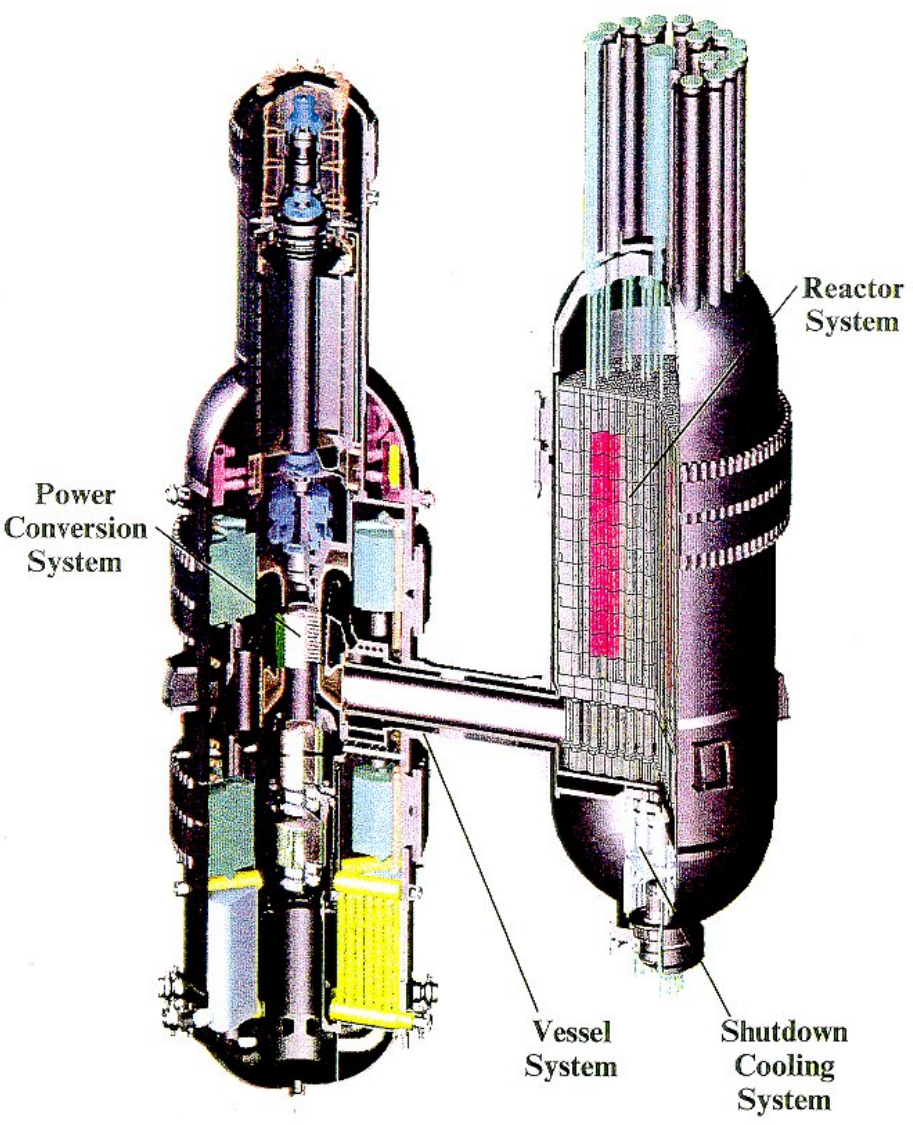

Fig. 3. Model of prismatic VHTR being developed for the Generation IV program. the HTR-PM.

\subsection{Liquid-Metal-Cooled Reactors}

Development of liquid-metal-cooled reactors (LMR) began shortly after the initial development of water-cooled reactors. The high thermal conductivity, high boiling points, and relatively low melting points of some liquid metals offered potential benefits of high core power density, small core volumes, and thin-walled pressure vessels compared to high-pressure LWRs. Candidate liquid metals that have been studied for commercial nuclear power include sodium, lead, and lead-bismuth. The coolants have low neutron moderation properties; therefore, the core contains a high fraction of fast neutrons, which yield higher numbers of neutrons per fission than do thermal neutrons. The additional neutrons can be used to breed fissile material from the fertile isotopes in the fuel-potentially extracting up to 60 times more energy from uranium than thermal reactors. 
A potential reduction in high-level waste repository requirements is also an attractive benefit of these reactors. The fast neutron spectrum in the core is favorable to transmuting plutonium and some other transuranic actinides into short-lived fission products to reduce or eliminate longlived, high-heat-producing radioactive waste in the spent nuclear fuel. Descriptions of current LMR concepts and technology assessments of potential future systems are provided in a number of compendium reports. ${ }^{16,17,18}$

Numerous SMR concepts with liquid-metal coolants have been developed ${ }^{7,8}$ and are mentioned in the following sections.

3.3.1. Sodium-Cooled Reactors: The anticipated rapid growth of the commercial nuclear power industry during its early days caused reactor designers to project an imminent shortfall in uranium supply. Consequently, experimental, prototype, and commercial-size liquid-metalcooled fast reactors using sodium or sodium-potassium coolants were planned and/or built to test fuels and materials and prove the technology. Sodium-based coolants have good nuclear and heat transfer properties and low corrosive effects on commonly used nuclear materials.

In the United States, the Advanced Liquid-Metal Reactor (ALMR) program in the 1980s and 1990s resulted in the design of the 160-MWe Power Reactor Inherently Safe Module (PRISM) sodium-cooled reactor. The PRISM design was one of the first advanced reactor designs to employ significant use of passive safety features and was designed as a power module to be used in packs to form a large electrical-capacity power plant. After termination of the ALMR program, General Electric continued to develop the design, with a significant increase in its power rating, resulting in the 380-MWe Super-PRISM (S-PRISM) design. ${ }^{19}$ Sodium-cooled fast reactor technology is one of the reactor technologies being developed within the Generation IV program and has been selected for the role of the GNEP advanced recycle reactor (ARR). A model of this concept is shown in Fig. 4.

Toshiba's recent $4 \mathrm{~S}$ reactor ${ }^{8}$ is a sodiumcooled, uranium-metal-fueled small reactor design with electric power generation capability of 10-50 MWe and/or process heat supply. The reactor is expected to be attractive in remote areas where the cost of alternative fossil-fuels for power generation is high. The reactor expects to achieve standardization through shop fabrication and be lightweight, compact, and transportable; passively safe; and seismically isolated. The reactor vessel is sealed for the expected 30year life of the system with no refueling required. The reactor design minimizes the use of active components and control systems and uses an electro-magnetic pump to eliminate a pump with rotating parts. The town of Galena, Alaska, has expressed

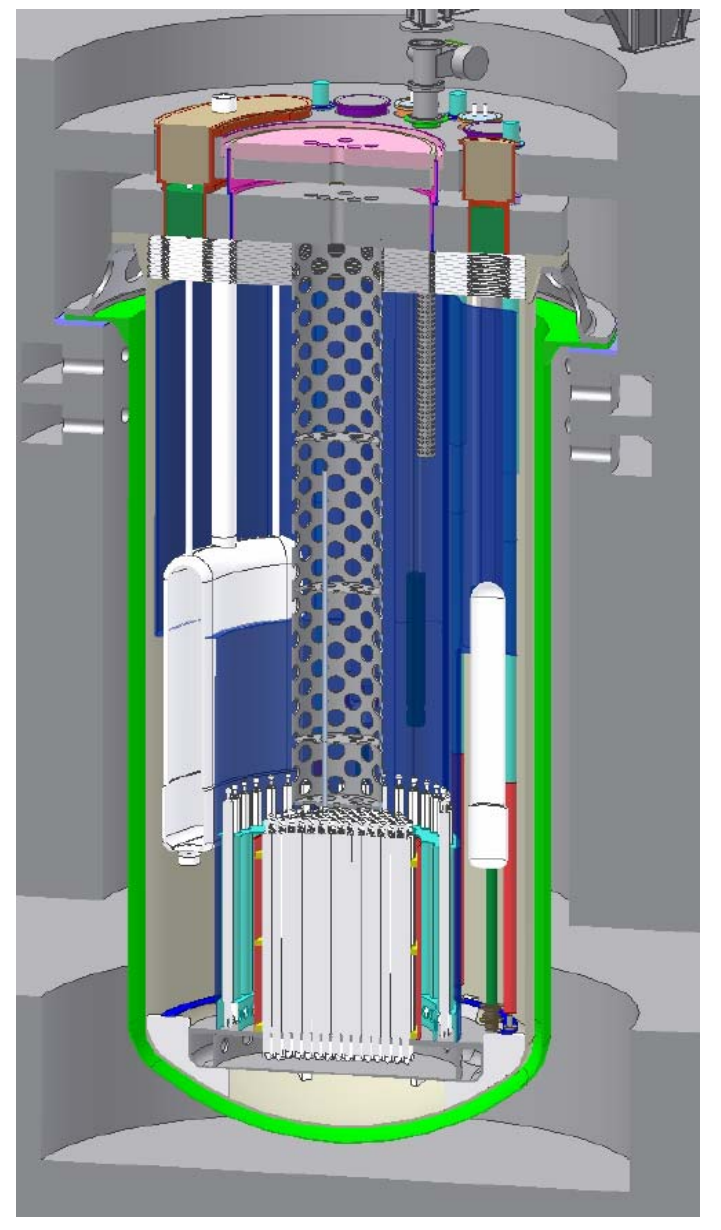

Fig. 4. Model of sodium-cooled ARR concept. 
interest in this system as a replacement or supplement to their costly diesel-fueled power generation plant.

3.3.2 Lead-Cooled Reactors: Relative to sodium coolant, lead and lead-bismuth coolants have a number of advantages such as higher boiling temperatures, low coolant void reactivity feedback, and low chemical reactivity with air, water, and steam. Disadvantages are that they are corrosive to reactor structural materials, especially at higher temperatures, and lead-bismuth produces ${ }^{210} \mathrm{Po}$ as an activation product, which presents a significant radiation hazard.

Liquid-metal-cooled reactors with lead-bismuth coolant were used in the 1960s and 1970s for Soviet submarines. Building on this experience, Russia is currently developing the lead-bismuthcooled SVBR-75/100. Each module produces between 75-100 MWe. The design reflects improvements in coolant technology and reactor materials, including corrosion and erosion control, radiation hazards particular to the coolant $\left({ }^{210} \mathrm{Po}\right)$, design and operation of lower speed primary coolant pumps, and handling coolant freeze/thaw cycles. The SVBR-75/100 also is designed to have an extended life core with whole-core refueling. The design incorporates passive decay heat removal, a simplified system design, closed-fuel-cycle compatibility, and safeguards/security provisions.

There is an ongoing project in Europe to develop a European Lead-cooled System (ELSY), which is a 600-MWe medium-sized reactor concept. The approach taken in the ELSY design is to use pure lead as the coolant and relying on well-understood and proven materials, technologies, and temperature-pressure conditions. This strategy is intended to ensure the ability to build and operate such a system with a high level of confidence.

In the United States, the SSTAR reactor ${ }^{20}$ is a lead-cooled small reactor (10-100 MWe) concept being developed by a team of national laboratories (Argonne, Lawrence Livermore, and Los Alamos) and the University of California at Berkeley (Fig. 5). Like the sodium-cooled 4S, this reactor is envisioned for limited power grids typical of developing countries or remote locations. Its output options include process heat for district heating, water desalination, or other purposes. This concept places an emphasis on safeguards and security, with the reactor core to remain sealed for approximately 15-20 years of operation using MOX or nitride fuel and then replaced with another complete core. Onsite access to the reactor core would be strictly limited by the long refueling period and the sealed core. The reactor is intended for autonomous, load-following operation, requiring only monitoring and security/protective services. The reactor would be factory made in transportable modules for site assembly. Features of the reactor include a natural circulation primary system, low primary system pressure, low power density, passive safety systems, compatibility with a closed fuel cycle, high power conversion efficiency, and a competitive price. Other reactor technologies related to the SSTAR concept are the lead-cooled STAR-LM concept and the lead-bismuth-cooled Encapsulated Nuclear Heat Source (ENHS) concept.

\subsection{Molten-Salt-Cooled Reactors}

In the 1960s and 1970s, a completely different reactor type was designed and developed in the United States, including the construction of two experimental reactors. This reactor type used a molten fuel-salt mixture circulated through a graphite moderator block to achieve a very compact, high-power reactor system. Although originally intended for aircraft propulsion applications, which were abandoned in the early 1960s, the technology for molten salt reactors (MSR) continued to be developed for a number of years. 


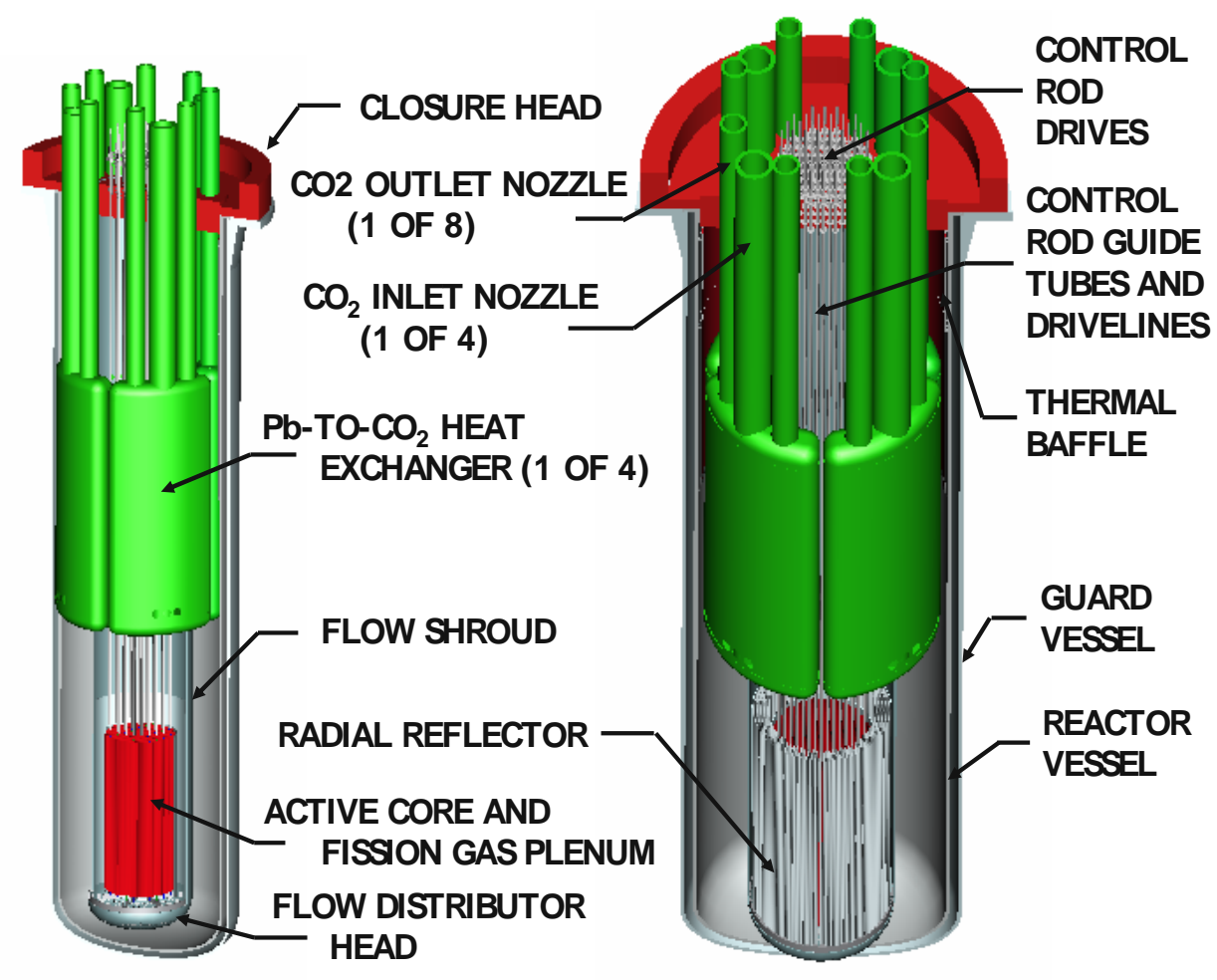

Fig. 5. Model of SSTAR lead-cooled reactor concept.

Today, a number of countries are studying MSRs for potential commercial power or hightemperature process heat applications. The primary motivation for considering the new technology is that plant electric-generating efficiencies increase as a function of the temperature of the turbine inlet temperature, ranging from roughly 33\% at typical LWR temperatures using a steam Rankine cycle to more than $50 \%$ at temperatures above $800^{\circ} \mathrm{C}$ using a gas Brayton cycle. Water-cooled reactors have a practical limit of about $350^{\circ} \mathrm{C}$ maximum temperature, and sodiumcooled reactors are typically designed for $500-600^{\circ} \mathrm{C}$. To achieve higher temperatures, designers typically switch to gas coolants to avoid phase-change issues with the liquid coolants. Moltensalt-cooled reactors offer opportunities to increase primary system temperature above that available from LWRs and sodium-cooled fast reactors (SFR) while offering benefits of liquid coolants such as good heat transfer characteristics and low primary system pressure.

Additionally, liquid salts are transparent, which improves inspection and maintenance operations relative to liquid metals, and the very high temperatures possible with molten salt reactors enable them to be used for industrial process heat applications such as the high-temperature production of hydrogen.

The earlier MSRs were fueled with uranium or thorium fluorides dissolved in molten lithium and beryllium fluoride salts. Because the fuel was liquid, fission products could be removed from the fuel while the reactor operated and fresh fuel could be added to the reactor as needed. Systems for the chemical control of the molten salts were developed, but this technology has been stagnant for more than 30 years. Recently, a new variant of the MSR has been studied that uses pure molten fluoride salts, that is, salt without fuel, to cool a graphite-moderated core containing standard graphite-coated particle fuel. This concept is referred to as the liquid-salt-cooled Advanced High Temperature Reactor (AHTR) and takes advantage of the excellent thermodynamic characteristics of the liquid salt to overcome many of the engineering challenges of the highpressure, high-temperature gas-cooled reactors. The MSR and the AHTR are described below. 
3.4.1 Molten-Salt-Fueled Reactors. A high-temperature $\left(\sim 1,400^{\circ} \mathrm{C}\right)$ MSR concept was identified by the Generation IV International Forum as a candidate for cooperative development. The concept has several advantages and disadvantages. ${ }^{21}$ Two major advantages are that it yields a high thermal efficiency and its low pressure primary system has low stored energy. Also, the salts do not readily release fission products and actinides, and because it is capable of on-line refueling, there is very little excess reactivity. Fuel is drained to passively cooled, criticality-safe dump tanks in emergencies. Disadvantages include the materials development requirements for the high-temperature operation, the necessity for remote maintenance of the radioactive primary system and intermediate system components, the capturing of volatile fission products, and purification of the fuel salt.

3.4.2 Molten-Salt-Cooled Reactors. In contrast to the MSR, the AHTR ${ }^{22}$ uses clean molten salt as a coolant and a solid, coated-particle fuel form identical to the prismatic block gas-cooled reactor. Coolant core exit temperatures may range from 700 to $1,000^{\circ} \mathrm{C}$. Although existing materials are qualified for the lower end of this temperature range, higher temperatures will require materials development activities. Unlike the gas reactor, the primary system pressure is low, thus reducing the required pressure vessel requirements. The high coolant heat transfer capabilities support higher volumetric core heat-generation rates (higher power per unit core volume) and also facilitate passive decay heat removal systems even for systems up to 1,200 MWe. A diagram of the current layout of the AHTR is shown in Fig. 6. A key advantage of the AHTR relative to gas-cooled reactors is the ability to achieve high-power outputs while retaining the passive safety performance of the graphite-moderated, particle-fuel core. An initial concept for a medium-sized version of the reactor for PRIMR applications is in the early stages of development in the United States.



Fig. 6. Diagram of molten-salt-cooled AHTR concept. 


\section{Evaluation of Technology Maturity for Near-Term Deployment}

As discussed in Section 2, there are a number of requirements that candidate PRIMR designs must meet in order to be considered for deployment to developing countries, including power output, plant safety, proliferation risk, safeguards, economics, and design maturity. Section 3 briefly described each of the four primary reactor coolant technology types (water, gas, liquidmetal, and molten-salt) and presented examples of specific designs that may be relevant to GNEP goals for grid-appropriate reactors. In this section, the operating experiences of the four reactor types are discussed in order to evaluate which technologies are viable candidates for a near-term PRIMR design. The emphasis in the descriptions given below is technology maturity, since this is expected to be a key discriminator among the technology choices. Note is also given to operational experience that impacts other requirements such as safety or proliferation risk, although these characteristics are primarily a function of the specific reactor design and engineering rather than the basic coolant technology.

\subsection{Water-Cooled Reactors}

Currently there are 435 commercial nuclear power reactors in service in 31 countries. ${ }^{23}$ Roughly 95\% of them are water-cooled, including 264 PWRs, 93 BWRs, 42 PHWRs, and 16 LWGRs. The remaining 5\% include 18 gas-cooled reactors and 2 sodium-cooled reactors. Additionally, there are approximately 160 naval ships powered by water-cooled reactors, with many more operational during the Cold War. Collectively, water-cooled reactors represent a technology with about 23,000 reactor-years of operational experience. Materials and operational issues are well known worldwide. Present-day commercial reactors perform reliably with average availability exceeding $90 \%$, have proven to be safe, generate power at highly competitive rates, and have long lifetimes (40-60 years). Of the approximately 30 reactors currently being constructed worldwide, over $90 \%$ are water cooled.

There have been two significant accidents at commercial LWRs: the Three Mile Island Unit 2 accident in 1979 and the Chernobyl Unit 4 accident in 1986. The TMI accident, which occurred in a loop-type PWR, was a result of several mechanical, electrical, and human factor errors. Although there were no direct deaths or injuries due to the accident, it was a huge financial loss for the owner. As a result, significant changes were made to plant design, regulation, and operation of similar PWRs in subsequent years.

The reactor accident in Chernobyl, which involved one of the 16 LWGRs, resulted in 31 immediate deaths, numerous injuries, and the release of massive amounts of radioactivity over an extended region. The accident occurred due to operator errors, design and control deficiencies, and lack of a full sealed containment building-a requirement for modern LWRs with conventional fuel designs. Some of the remaining LWGRs have been shutdown because of safety concerns, and many of the remaining units are programmed for replacement with an entirely different type of reactor design. In addition to safety concerns, the on-line refueling feature of the LWGRs introduces a proliferation concern due to increased opportunities for access to fuel and misuse of the reactor for the production of weapon-usable material.

The PHWR-type CANDU reactors have an excellent safety record. They have design features that enable them to be refueled at power, thus avoiding refueling outages required by other reactor designs and consequently increasing the availability of the plant; however, similar to LGWRs, they introduce a proliferation concern due to the increased fuel access and material production opportunities. 
Although LWRs initially had very low plant availability, and hence poor cost competitiveness, current plants in the United States and worldwide have availabilities generally exceeding $90 \%$ and frequently provide electricity at costs that are below competitive sources.

There is a broad vendor, service, and supply base for water-cooled reactors. Owner groups and industry groups share information worldwide to help ensure safe, economic plant operation. Regulators in these countries also are experienced with water-cooled reactor designs, construction monitoring, operational issues, fuel performance issues, operator training and licensing, safety assessment, and domestic safeguards/security requirements. These regulators also interact with each other worldwide. Additionally, IAEA approaches to international safeguards for LWRs are well understood. Thus, LWR maturity applies to design; construction; performance; supply of materials, fuels, and components; operations; service; regulatory; and safeguarding aspects of water-cooled reactors. No equivalent level of maturity exists with any other reactor technology.

\subsection{Gas-Cooled Reactors}

The first gas-cooled commercial power reactor, the Calder Hall Nuclear Power Station in the United Kingdom, began operation in 1956. There are currently 18 commercial GCRs in operation, all in the U.K., with an average output of about 540 MWe. Thirty-eight GCRs that were previously operational in France, Germany, Italy, Japan, Spain, and the United States have been decommissioned. Although GCRs were the predominant reactor type in Europe during the 1960s, especially the U.K. and France, the gas-cooled reactor was replaced by the PWR as the reactor design of choice in France by the 1970s. Gas-cooled reactors continued to be built in the U.K. through the 1980s; however, the most recent plant to be built there was also a PWR.

There are approximately 1,600 reactor-years of experience with GCRs, ranking them second to water-cooled reactors in terms of total operating experience. Including both operating and decommissioned plants, the average operating lifetime per plant is just under 30 years. The U.K. plants averaged about 35 years of operation, while plants outside the U.K. averaged about 17 years. The two U.S. plants, Peach Bottom 1 and Fort St. Vrain, operated for 7 years and 13 years, respectively.

Although many aspects of the Fort St. Vrain plant worked well, there were a number of persistent operational difficulties. ${ }^{24}$ Safety consequences of moisture intrusion, particularly low amounts of moisture over extended periods of time, were an important issue. Moisture degraded both the control rod drives and reserve shutdown systems such that multiple rods failed to scram as required during a 1984 event. Helium leakage events were noted twice. There were also some problems with corrosion of the pre-stressed concrete reactor vessel tendons and some superficial cracking of fuel element webs. However, except for the problems with the reactor shutdown system, no undue safety hazards were noted.

The water-lubricated circulator bearings were determined to be the primary source of the moisture ingress and, in retrospect, were not a good design choice. ${ }^{25}$ However, the coated particle fuel and reactor core performed well. Worker exposures were noted to be about $1 \%$ that of workers at water-cooled reactors. The thermal efficiency of the 330-MWe (842-MWt) plant was about $20 \%$ higher than LWRs of similar vintage.

\subsection{Liquid-Metal-Cooled Reactors}

Although LMRs were envisioned in the 1960s to play a large future role in the nuclear electricity generation mix, concerns regarding proliferation risk, safety, and economics curtailed work on fast reactor systems in the United States. Additionally, new uranium deposits were discovered and electricity demand growth dropped significantly following the oil embargoes of the 1970s. In the late 1970s, the United States chose to forego nuclear fuel reprocessing because of 
proliferation concerns. The Three Mile Island 2 accident in 1979 raised questions about nuclear plant safety and resulted in operating plant shutdowns, new plant delays, and future plant cancellations with major financial impacts to utilities and rate payers. These collective factors severely constrained additional nuclear plant developments in the United States, and similarly affected the industry worldwide. Consequently, the compelling case for breeder reactors was severely diminished. The sodium-cooled Clinch River Breeder Reactor demonstration project, started in the mid-1970s, was cancelled in 1983. Research and development activities on U.S. LMRs were cut significantly and sodium-cooled research facilities such as EBR-II and FFTF were ultimately shutdown.

A significant knowledge base still remains for sodium-cooled reactors and research

accomplishments are well documented. It remains a viable option, although the U.S. design, fuel, materials, manufacturing, construction, operations, service, and regulatory infrastructures have eroded significantly for this technology. Based on the relative maturity of sodium-cooled reactor technology compared to other fast-spectrum reactor options, the SFR has been selected as the preferred fast reactor technology for the Generation IV Program ${ }^{26}$ and is the technology to be used for the GNEP ARR.

A total of nine SFRs were built and used for commercial power generation. ${ }^{3}$ These units collectively had 138 years of operational experience through the end of 2006. Three more reactors generated electricity for on-site use and add another 95 years of cumulative operational experience. By including other experimental, research, or non-power-producing reactors, the total operational SFR experience is about 300 reactor-years.

Prototype and commercial sodium-cooled reactors have been operated generally well in France, Japan, Russia, and Kazakhstan. These LMRs almost universally used oxide fuels-uranium and plutonium. Exceptions to this were the Dounreay (U.K.), EBR-II (U.S.), and Fermi 1 (U.S.) experimental reactors, which used metal fuel. The uranium oxide fuel infrastructure is mature worldwide; the plutonium or mixed-oxide fuel fabrication infrastructure for commercial fuel is well developed in France and more recently in Japan.

The Phenix reactor (France), Dounreay experimental and prototype reactors (U.K.), BN-350 (Kazakhstan), BN-600 reactor (Russia), and JOYO experimental reactor (Japan) have had lengthy operating histories. All are sodium cooled except the Dounreay experimental reactor, which was cooled by sodium-potassium. Based on the experience in France, Germany, and the U.K., a European fast reactor design was completed to the point of producing a reliable cost estimate. ${ }^{27}$ Thus, the SFR technology may be considered most mature in Russia, France, and Japan.

Unfortunately, there have been difficulties transitioning from experimental and demonstration reactors to commercial-sized SFR plants. The Super-Phenix reactor (France), a 1200-MWe commercial-size plant, had problems with balance-of-plant issues and building structures. The plant also experienced some sodium leakage and associated chemical reactions. Anti-nuclear protests ultimately led to government actions to permanently shutdown the plant. The prototype Monju reactor (Japan) operated for several months following its startup in 1995 until a sodium leak in the secondary system forced an extended shutdown lasting several years. Estimates of the cost of electricity from commercial sodium-cooled reactors range from 20-50\% higher than modern LWRs.

In the case of lead- and lead-bismuth-cooled reactors, there are about 80 reactor-years of accumulated operational experience based entirely on 12 Russian submarine and land-based reactors. The longest operating unit ran for approximately 10 years. Their initial experience, based in part on fielding the technology before appropriate oxygen control systems were included, identified significant coolant and materials compatibility challenges, which have been the subject of considerable follow-on research in Russia and elsewhere. For example, if coolant 
conditions are not carefully controlled, coolant oxidation and corrosion products can build up in the coolant and were observed to lead to flow blockages in the coolant channels. These conditions led to core damage of an early navy propulsion reactor. ${ }^{28}$ Subsequently, Russian scientists gained valuable experience in managing corrosion through materials development and improved chemical control and purity of the coolant systems. ${ }^{29}$ They also developed methods to minimize the radiation hazard from ${ }^{210}$ Po produced in the lead-bismuth coolant. Because no commercial or large-scale lead-cooled reactors have been operated, it is difficult to predict the cost performance of this type of reactor relative to other reactor types.

\subsection{Molten-Salt-Cooled Reactors}

Numerous test loops were operated to qualify suitable materials to contain the molten fuel-salt mixture in the primary system and pure molten salt mixtures in the secondary heat transport systems of early MSRs. The Aircraft Reactor Experiment (ARE), which was a 2.5-MWt experimental MSR, operated only briefly due to a number of operational problems. The ARE was followed by the Molten Salt Reactor Experiment (MSRE), a larger 8-MWt experimental reactor that ran for nearly 4 years before being shutdown at the end of the MSR program. Initially, the salt-fuel mixtures were seen to cause significant corrosion of the metallic container materials. One specific issue was the leaching of chromium from the metals in the hottest parts of the system and a corresponding deposition of chromium in the colder regions. By the end of the MSR program, special low-chromium alloys had been developed, tested, and code qualified for use with the molten salts up to $750^{\circ} \mathrm{C}$. The accumulated experience with these early reactors and the extensive testing program is documented in over 1000 technical reports.

Although some aspects of the technology are shared with other reactor types, the salt chemistry control and cleanup systems are unique to the MSR and the associated technology infrastructure is nonexistent today. Also, because of the liquid, circulating fuel characteristic of the MSR, licensing of the reactor will be considerably longer than that for traditional stationary fuel reactors. In the case of the liquid-salt-cooled AHTR, it shares considerable technology with hightemperature helium-cooled reactors and will be more easily licensed than the MSR. However, most of the salt chemistry cleanup and control technology will need to be reestablished. Also a low-power test reactor, and perhaps an intermediate-power prototype reactor will be needed prior to commercial deployment. As with lead-cooled reactors, there is insufficient experience to reliably predict the economic performance of molten salt or liquid-salt-cooled reactors.

\section{Summary and Conclusions}

Near-term PRIMR deployment to developing countries is needed to meet the current and rapidly growing demand for energy in several countries, while also providing the United States with the opportunity to influence how that demand is met and enable U.S. industry to compete effectively in the global nuclear market. However, it is evident from the discussions in the previous section that some technologies will require significantly more development and engineering work and more operational experience to be considered for commercial applications and, therefore, are not suitable for near-term consideration.

It is also evident that the selection of a PRIMR system, whether for the near term or longer term, must consider more than basic reactor coolant technology-design choices dramatically impact the overall safety and proliferation risk of the plant. For example, batch-refueled, loop-type PWRs have been demonstrated to be acceptably safe, but the continuously refueled, channel-type LWGRs, which fall within the same category of water-cooled reactors, pose significant safety and 
proliferation risk concerns. Hence specific design implementation of a reactor technology is also of paramount importance, and the selection of reactors for ultimate deployment will require a comprehensive assessment of specific candidate designs relative to quantitative criteria derived from the full set of requirements. Efforts are under way in the United States and with our international partners to develop a suitable set of requirements and criteria that will be used to select a near-term design and also provide a focus for the development of improved technologies for longer-term PRIMR designs.

The purpose of this trade study was to identify the most promising reactor technology for nearterm deployment. Therefore, it is appropriate to focus on technology maturity as the fundamental discriminator. Based on the discussions in Section 4, a summary of key maturity factors is given in Table 2. The following points help to explain and clarify the data given in the table:

- The values for "Operational Experience" are estimates of cumulative operating times and include the operation of experimental, test, prototype, and commercial reactors. It is worth noting that not all of the experience was positive, as noted in preceding sections.

- The values for "Time for Final Design Approval” are judgments based on U.S. NRC experience with licensing reactors of the designated type. Actual times could be longer depending on the extent of new technologies or engineering employed in a specific design.

- The parameter "Existing Vendors" includes only companies that have sold previous reactors and are actively marketing current designs of the designated types. These include the following:

o Water: Westinghouse (AP1000, IRIS), General Electric (ABWR, ESBWR), Areva (EPR), Atomic Energy of Canada (CANDU-6), Mitsubishi (APWR), Russia (VVER-1000, KLT-40S), India (PHWR), Republic of Korea (KSNP, SMART), China (PWR)

o Gas: General Atomics (GT-MHR), Eskom (PBMR), Areva (VHTR), China (HTR-PM)

o Sodium: General Electric (S-PRISM), Russia (BN-800), Toshiba (4S),

o Lead: Russia (SVBR-75/100)

Table 2. Maturity indicators for primary reactor types

\begin{tabular}{|l|c|c|c|c|c|}
\hline \multirow{2}{*}{ Maturity Parameter } & \multicolumn{5}{|c|}{ Coolant } \\
\cline { 2 - 6 } & Water & Gas & Sodium & Lead & Salt \\
\hline \hline $\begin{array}{l}\text { Operational Experience } \\
\text { (reactor-years) }\end{array}$ & 23,000 & 1,600 & 300 & 80 & 4 \\
\hline $\begin{array}{l}\text { Time for Final Design Approval } \\
\text { (years) }\end{array}$ & 3 & 5 & 5 & $>5$ & $>5$ \\
\hline Existing Vendors & 9 & 4 & 3 & 1 & 0 \\
\hline Pre-Commercial Demonstration & No & Yes & Yes & Yes & Yes \\
\hline
\end{tabular}


- Although somewhat subjective, the need for a "Pre-Commercial Demonstration" unit is assumed to be needed for all systems other than water-cooled reactors to facilitate licensing. The same may be true also for a water-cooled design if its design engineering is significantly different from currently licensed reactors.

Based on these figures, light water technology is the clear choice for the near-term GNEP gridappropriate reactor because it is overwhelmingly the most mature reactor technology with the highest level of industrial and regulatory support needed for deployment in the near-term. As stated above, the next step will be to evaluate specific water-cooled reactor designs relative to several requirements that are under development, including proliferation risk, safety, security, and economics. 


\section{References}

1. The National Security Strategy of the United States of America (March, 16, 2006): 29.

2. “Russia to Build World’s First Floating Nuclear Power Station,” MOSNews, Sept. 9, 2005.

3. “New Units At Home, New Opportunities Abroad,” World Nuclear News, Feb. 27, 2007.

4. "An Analysis of the Role of the Small and Medium Reactors Program,” TechSource, Inc., March 2007.

5. "Potential of Small and Medium Sized Reactors (SMR) to Support the GNEP Vision," Advanced Systems Technology and Management, Inc., November 2006.

6. Global Database of Operational Generation Plants, Edition 3, 2006, ABS Energy Research, August 2007.

7. Evaluation Methodology for Proliferation Resistance and Physical Protection of Generation IV Nuclear Energy Systems, Revision 5, Generation IV International Forum, GIF/PRPPWG/2006/005, November 30, 2006.

8. The Pysical Protection of Nuclear Material and Nuclear Facilities, International Atomic Energy Agency, INFCIRC/225, Rev. 4 (corrected).

9. Status of Innovative Small and Medium Sized Reactor Designs 2005: Reactors with Conventional Refueling Schemes, International Atomic Energy Agency, IAEA-TECDOC1485, March 2006.

10. Status of Innovative Small and Medium Sized Reactor Designs 2006: Reactors without Onsite Refueling, International Atomic Energy Agency, IAEA-TECDOC-1536, January 2007.

11. Generation IV Roadmap: Description of Candidate Water-Cooled Reactor Systems Report, Issued by the Nuclear Energy Research Advisory Committee and the Generation IV International Forum, GIF-015-00, December 2002.

12. M.D. Carelli and B. Petrovic, "IRIS-Progressing Toward International Deployment," Proceedings of ICAPP-06, Reno, NV, June 4-8, 2006.

13. Status of advanced light water reactor designs 2004, International Atomic Energy Agency, IAEA-TECDOC-1391, May 2004.

14. Design and development status of small and medium reactor systems 1995, International Atomic Energy Agency, IAEA-TECDOC-881, May 1996.

15. http://www.eskom.co.za/nuclear_energy/pebble_bed/pebble_bed.html.

16. Fast Reactor Database, International Atomic Energy Agency, IAEA TECDOC-866, February 1996. 
17. Generation IV Roadmap—R\&D Scope Report for Liquid-Metal-Cooled Reactor Systems, Nuclear Energy Research Advisory Committee and the Generation IV International Forum, GIF-005-00, December 2002.

18. Generation IV Roadmap-Description of Candidate Liquid-Metal-Cooled Reactor Systems Report, Nuclear Energy Research Advisory Committee and the Generation IV International Forum, GIF-017-00, December 2002.

19. C. E. Boardman et al., "Optimizing the Size of the Super-PRISM Reactor,” Proceedings of ICONE-8, Baltimore, MD, April 2-6, 2000.

20. J. J. Sienicki et al., Status Report on the Small Secure transportable Autonomous Reactor (SSTAR)/Lead-Cooled Fast Reactor (LFR) and Supporting Research and Development, ANL-GenIV-089, September 2006.

21. C. W. Forsberg, "Safety and Licensing Aspects of the Molten Salt Reactor," American Nuclear Society Annual Meeting, Pittsburg, PA, June 2004.

22. D. T. Ingersoll et al., Trade Studies on the Liquid-Salt-Cooled Very High-Temperature Reactor: Fiscal Year 2006 Progress Report, ORNL/TM-2006/140, February 2007.

23. IAEA Power Reactor Information System, http://www.iaea.org/programmes/a2/.

24. Fort Saint Vrain Gas Cooled Reactor Operational Experience. Oak Ridge National Laboratory, NUREG/CR-6839, January 2004.

25. http://gt-mhr.ga.com/gcr all.html.

26. The U.S. Generation IV Fast Reactor Strategy, U.S. Department of Energy, DOE/NE-0130, October 2006.

27. Comparative assessment of thermophysical and thermohydraulic characteristics of lead, leadbismuth and sodium coolants for fast reactors, International Atomic Energy Agency, IEAETECDOC-1289, June 2002.

28. A. V. Zrodnikov et. al., "Heavy liquid metal coolant - lead-bismuth and lead - technology," Atomic Energy, Vol. 97, No. 2, 2004.

29. B. F. Gromov et al., "Use of lead-bismuth coolant in nuclear reactors and accelerator-driven systems,” Nuclear Engineering and Design, Vol. 173, pp. 207-217, 1997. 